\title{
New Signal Constellation Pairs for the ZTM-OFDM-IM System
}

\author{
Shuang $\mathrm{Li}^{1}$ and Seog Geun Kang $\mathbb{D}^{2}$ \\ ${ }^{1}$ Department of Electrical and Electronic Engineering, Gyeongsang National University, Jinju, Republic of Korea \\ ${ }^{2}$ Department of Semiconductor Engineering and the Engineering Research Institute, Gyeongsang National University, \\ Jinju, Republic of Korea \\ Correspondence should be addressed to Seog Geun Kang; sgkang@gnu.ac.kr
}

Received 23 June 2020; Revised 2 July 2020; Accepted 11 July 2020; Published 1 August 2020

Academic Editor: KI-IL Kim

Copyright (C) 2020 Shuang Li and Seog Geun Kang. This is an open access article distributed under the Creative Commons Attribution License, which permits unrestricted use, distribution, and reproduction in any medium, provided the original work is properly cited.

\begin{abstract}
In this paper, we investigate new signal constellation pairs for mapping active subcarriers of the zero-padded trimode orthogonal frequency division multiplexing with index modulation (ZTM-OFDM-IM) systems. In the presented system, one of a constellation pair is the same as the one used in the previous work, and the other is a constellation larger than the one used in the previous work. It increases the minimum Euclidean distance between the subblocks of the ZTM-OFDM-IM system with new constellation pairs under the constraint of the same spectral efficiency. Computer simulation in AWGN and frequency-selective fading channels shows that the new ZTM-OFDM-IM system has a much lower bit error rate than OFDM-IM and dual-mode OFDM-IM and slightly outperforms the system with conventional constellation pairs. Since the proposed constellation pairs prove the error performance improvement of the system, it is considered that a further study on generalized design of the constellation pair for the ZTM-OFDM-IM system is necessary in the future.
\end{abstract}

\section{Introduction}

Due to its large capacity, high spectral efficiency, and robustness against frequency-selective fading [1], orthogonal frequency division multiplexing (OFDM), a multicarrier modulation technique, has been widely adopted in various wireless communication systems and standards such as wireless fidelity (Wi-Fi) [2], long-term evolution (LTE) [3], and world interoperability for microwave access (WiMAX) [4] for next-generation wireless communications. It is also an attractive advantage of an OFDM system for wireless communications to effectively eliminate intersymbol interference (ISI) by inserting a cyclic prefix (CP) between successive symbols [5].

To accommodate the requirements for high spectral and energy efficiencies, a lot of studies on index modulation (IM) have been carried out. In the IM scheme, a part of indexed resources are activated and used for data transmission [6, 7]. A subcarrier-index modulated (SIM) OFDM [8] and an enhanced SIM-OFDM (ESIM-OFDM) scheme [9] introduce the concept of IM into the frequency domain to achieve diver- sity gain. However, the spectral efficiency of both systems is very low compared to the conventional OFDM scheme. To mitigate this problem, OFDM with IM (OFDM-IM) has been proposed to flexibly transmit IM bits by controlling the index of active subcarriers $[10,11]$. In the OFDM-IM system, information bits are divided into symbol bits and index bits. The former are mapped to the activated subcarriers in the same way as the traditional OFDM modulation, and the latter indicate the pattern of the activated subcarriers in the OFDM subblock. Hence, the energy efficiency of the scheme can be significantly improved, while the spectral efficiency is limited [12]. To improve the spectral efficiency of the OFDM-IM system, dual-mode OFDM-IM (DM-OFDM-IM) has been proposed, where all subcarriers of a subblock are activated and mapped with two different constellations [13]. As the frequency spectrum can be fully utilized while maintaining the characteristics of the IM scheme, the DM-OFDM-IM generally outperforms the OFDM-IM. Though the DMOFDM-IM scheme has a little loss of energy efficiency, it can enhance the data rate more than the OFDM-IM. 
As a compromise between spectral efficiency and energy efficiency, the zero-padded trimode OFDM-IM (ZTMOFDM-IM) system has been proposed in [14]. Here, the subcarriers are divided into several disjoint subblocks. A part of the subcarriers in each subblock is modulated by two distinguished constellations, and the other subcarriers are left blank, so that energy efficiency of the system can be increased. To avoid decreasing spectral efficiency, a larger constellation is usually exploited to map the active subcarriers.

In this paper, we introduce new constellation pairs to improve error performance of the ZTM-OFDM-IM system. Here, one of the two mappers used to map active subcarriers uses the same signal constellation as the one used in the previous work, while the other uses a larger constellation than the one used in [14]. This increases the minimum Euclidean distance (MED) between the subblocks of the ZTM-OFDMIM system with new constellation pairs under the constraints of the same spectral efficiency. The proposed constellation pairs demonstrate the possibility of improving error performance of the system.

The rest of this paper is organized as follows. The transceiver models of the OFDM systems with the IM scheme are briefly introduced in Section II. In Section III, the new pairs of constellations are discussed in detail. Performance of the proposed ZTM-OFDM-IM system is analyzed in Section IV. Finally, some conclusions are provided in Section V.

\section{The OFDM Systems with Index Modulation}

There are three typical index modulation schemes for the OFDM system. The OFDM-IM and DM-OFDM-IM can be considered as special cases of the ZTM-OFDM-IM system. The typical transmitter structure of the ZTM-OFDM-IM is shown in Figure 1, where S/P and P/S represent serial-toparallel and parallel-to-serial converters, respectively. IFFT is the $N$-point inverse fast Fourier transform. For an OFDM signal with $N$ subcarriers $\mathbf{X}=[X(1), X(2), \cdots, X(N)]^{T}$ in the frequency domain, all subcarriers are divided into $G$ disjoint subblocks to represent the signal as $\mathbf{X}=\left[\mathbf{X}^{(1)}, \mathbf{X}^{(2)}, \cdots, \mathbf{X}^{(G)}\right]^{T}$, where $\mathbf{X}^{(g)}=\left[X_{1}^{(g)}, X_{2}^{(g)}, \cdots, X_{n}^{(g)}\right]^{T}, \quad g=1,2, \cdots, G$, is the subcarriers in the $g$ th subblock and $n=N / G$ is the number of subcarriers in a subblock. Thus, the OFDM signal can be rewritten as

$\mathbf{X}=\left[X_{1}^{(1)}, X_{2}^{(1)}, \cdots, X_{n}^{(1)}, X_{1}^{(2)}, X_{2}^{(2)}, \cdots, X_{n}^{(2)}, \cdots, X_{1}^{(G)}, X_{2}^{(G)}, \cdots, X_{n}^{(G)}\right]^{T}$

The $m$ input information bits are divided into $G$ parallel data streams of $p=m / G$ bits. $p=p_{M}+p_{I}$ information bits for a group are divided into $p_{I}$ index bits and $p_{M}$ symbol bits, which are fed to the index selector and a pair of constellations for mappers, respectively. $p_{A}$ from the $p_{M}$ symbol bits are for signal mapping using the mapper $A$, while the remaining $p_{B}=p_{M}-p_{A}$ bits are for the mapper $B$. Since the constellations for signal mapping should be distinguishable, the signal points of the constellation $\mathrm{A}$ and those of the constellation B are not overlapped with each other. To prevent the spectral efficiency of the system from being reduced, the constellation size $M_{B}$ for the mapper B is generally larger than $M_{A}$, where the constellation size implies the number of elements in the constellation.

According to the $p_{I}$ index bits, the index selector determines one of $2^{p_{I}}$ index patterns expressed as

$$
\mathbf{I}=\left[\mathbf{I}^{(1)}, \mathbf{I}^{(2)}, \cdots, \mathbf{I}^{\left(2^{p_{I}}\right)}\right]^{T}
$$

where the $i$ th pattern $\mathbf{I}^{(i)}$ indirectly transmits $p_{I}$ bits given as

$$
\boldsymbol{I}^{(i)}=\left[I^{(i)}(1), I^{(i)}(2), \cdots, I^{(i)}\left(p_{I}\right)\right]
$$

for $i=1,2, \cdots, 2^{p_{I}}$. With the fixed index pattern, there are $2^{p_{M}}$ possible transmission signals of

$$
\mathbf{S}_{\mathbf{I}^{(i)}}=\left[\mathbf{S}_{\mathbf{I}^{(i)}}^{(1)}, \mathbf{S}_{\mathbf{I}^{(i)}}^{(2)}, \cdots, \mathbf{S}_{I^{(i)}}^{\left(2^{p_{M}}\right)}\right]^{T} .
$$

Each $\mathbf{S}_{\mathbf{I}^{(i)}}^{(j)} \subset \mathbf{S}_{\mathbf{I}^{(i)}}$ composed of $n$ subcarrier signals can be represented as

$$
\mathbf{S}_{\mathbf{I}^{(i)}}^{(j)}=\left[\begin{array}{llll}
S_{\mathbf{I}^{(i)}}^{(j)}(1) & S_{\mathbf{I}^{(i)}}^{(j)}(2) & \cdots & S_{\mathbf{I}^{(i)}}^{(j)}(n)
\end{array}\right]
$$

for $j=1,2, \cdots, 2^{p_{M}}$. Hence, there are $2^{p}$ transmission signals for $\mathbf{X}^{(g)}$. In each subblock, $k_{A}$ active subcarriers from $n$ subcarriers are modulated by the mapper $\mathrm{A}$, and the $k_{B}=k-k_{A}$ active subcarriers from the remaining $\left(n-k_{A}\right)$ subcarriers are modulated by the mapper $\mathrm{B}$, where $k$ is the total number of active subcarriers. Since inactive subcarriers are also included in the transmitted OFDM signal, the number of inactive subcarriers $(n-k)$ should be greater than zero. Therefore, the numbers of bits $p_{A}$ and $p_{B}$ modulated by mapper $\mathrm{A}$ and mapper $\mathrm{B}$ are

$$
\begin{aligned}
& p_{A}=k_{A} \log _{2}\left(M_{A}\right), \\
& p_{B}=k_{B} \log _{2}\left(M_{B}\right),
\end{aligned}
$$

respectively. Then, $p_{M}$ symbol bits are

$$
p_{M}=k_{A} \log _{2}\left(M_{A}\right)+k_{B} \log _{2}\left(M_{B}\right) .
$$

The index selector uses $p_{I}$ of the $p$ input bits to divide the index for a subblock into two index subsets. The number of bits needed to select an appropriate index mode is

$$
p_{I}=\left\lfloor\log _{2}\left(\begin{array}{c}
n \\
k_{A}
\end{array}\right)+\log _{2}\left(\begin{array}{c}
n-k_{A} \\
k_{B}
\end{array}\right)\right\rfloor,
$$

where $\lfloor x\rfloor$ denotes the greatest integer that is not greater than or equal to $x$.

Except for the subcarrier indexing method, the OFDMIM and the DM-OFDM-IM have the same functional structures as the ZTM-OFDM-IM. In the OFDM-IM scheme, only one constellation is used to generate active subcarriers. 


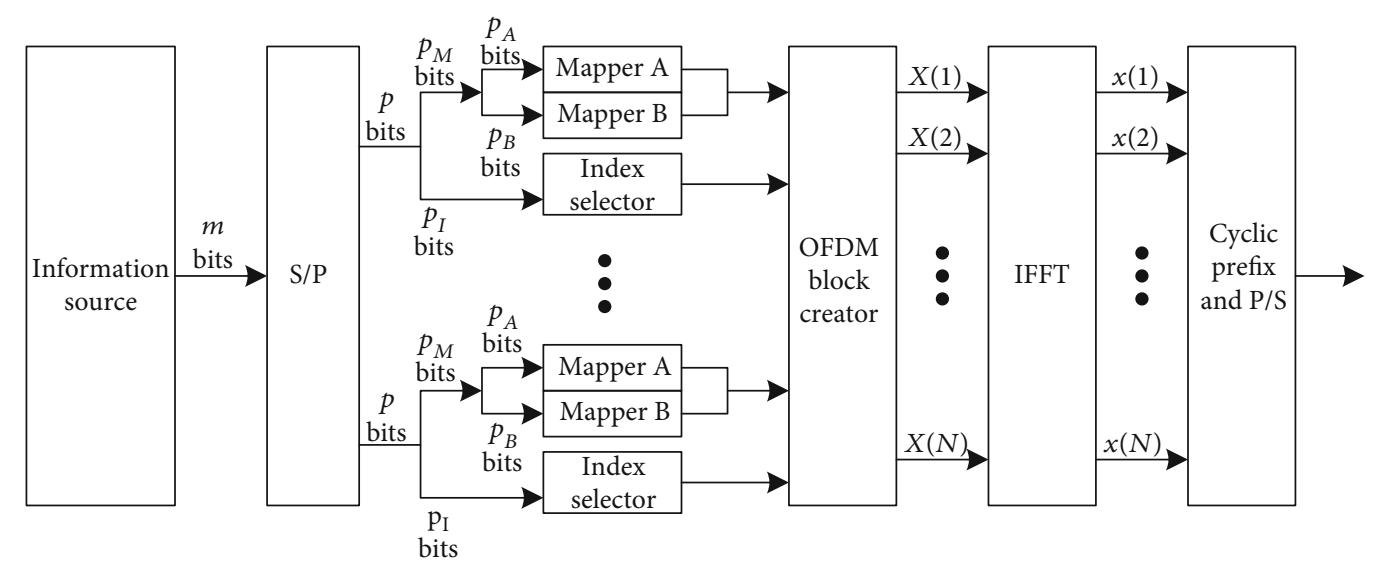

Figure 1: The transmitter structure of the ZTM-OFDM-IM system.

It is equivalent to the ZTM-OFDM-IM system with $k=k_{A}$, $k_{B}=0$, and $M_{B}=0$. Thus, it can be a special case of the ZTM-OFDM-IM with low spectral efficiency. The DMOFDM-IM scheme exploits a pair of constellations to generate the transmitted signals. However, it is different from the ZTM-OFDM-IM in that all subcarriers are activated for signal transmission, that is, $n-k=0$. Thus, it is considered as a ZTM-OFDM-IM system with low energy efficiency.

The OFDM system with an index modulation scheme also performs $N$-point IFFT to produce the signal $\mathbf{x}=$ $[x(1), x(2), \cdots, x(N)]^{T}$ in the time domain. To eliminate the adverse effect of ISI caused by the delay spread of multipath fading channel, a CP of length $L$ is added in front of the OFDM signal. Then, the spectral efficiency of the OFDM system is

$$
\eta=\frac{N p}{(N+L) n} .
$$

Suppose that the transmission environment is a frequencyselective Rayleigh fading channel of which the impulse response is $\mathbf{h}=[h(1), h(2), \cdots, h(v)]^{T}$ with the distribution of $\mathscr{C} \mathcal{N}(0,1 / v)$, where $v$ is the maximum delay spread. We assume that the length of CP should be greater than the maximum delay spread, that is, $L>v$ to completely eliminate the ISI. After removal of the $\mathrm{CP}$ and FFT operation in the receiver, the received OFDM signal in the frequency domain is $\mathbf{Y}=$ $[Y(1), Y(2), \cdots, Y(N)]^{T}$. Then, the received signal in the $g$ th subblock $\mathbf{Y}^{(g)}=\left[Y_{1}^{(g)}, Y_{2}^{(g)}, \cdots, Y_{n}^{(g)}\right]^{T}$ can be expressed as

$$
\mathbf{Y}^{(g)}=\operatorname{diag}\left\{\mathbf{X}^{(g)}\right\} \mathbf{H}^{(g)}+\mathbf{W}^{(g)}
$$

where diag $\left\{\mathbf{X}^{(g)}\right\}$ is an $n \times n$ diagonal matrix of which the main diagonal elements are $\mathbf{X}^{(g)} . \mathbf{W}^{(g)}=\left[W_{1}^{(g)}, W_{2}^{(g)}, \cdots, W_{n}^{(g)}\right]^{T}$ represents complex additive white Gaussian noise (AWGN) of $\mathscr{C} \mathscr{N}\left(0, N_{0}\right)$, that is, zero mean and variance of $N_{0} \cdot \mathbf{H}^{(g)}=$ $\left[H_{1}^{(g)}, H_{2}^{(g)}, \cdots, H_{n}^{(g)}\right]^{T}$ is a coefficient vector of the fading channel in the $g$ th subblock.
The maximum likelihood (ML) detector is used to minimize the Euclidean distance between the estimated subblocks and the received subblocks. The estimation of the $g$ th subblock can be determined by

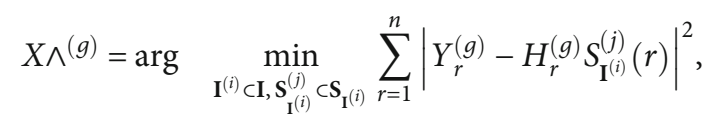

where $S_{\mathbf{I}^{(i)}}^{(j)}(r)$ represents the $j$ th transmission signal of the $r$ th subcarrier with the fixed index pattern $\mathbf{I}^{(i)}$. A simple look-up table can be used to recover the information bits from the subblocks corresponding to the transmitted symbols and index patterns.

\section{The Improved Constellation Pairs for ZTM- OFDM-IM}

When an ML detector is exploited, error performance of the ZTM-OFDM-IM system depends largely on the MED between different OFDM subblocks. The Euclidean distance between two subblocks can be defined as

$$
D\left(\mathbf{S}_{\mathbf{I}^{\left(i_{1}\right)}}^{\left(j_{1}\right)}, \mathbf{S}_{\mathbf{I}^{\left(i_{2}\right)}}^{\left(j_{2}\right)}\right)=\sqrt{\sum_{r=1}^{n}\left|S_{\mathbf{I}^{\left(i_{1}\right)}}^{\left(j_{1}\right)}(r)-S_{\mathbf{I}^{\left(i_{2}\right)}}^{\left(j_{2}\right)}(r)\right|^{2}}
$$

where $S_{\mathbf{I}^{\left(i_{1}\right)}}^{\left(j_{1}\right)}(r), S_{\mathbf{I}^{\left(i_{2}\right)}}^{\left(j_{2}\right)}(r) \in \mathbf{S}$ and $\mathbf{I}^{\left(i_{1}\right)}, \mathbf{I}^{\left(i_{2}\right)} \subset \mathbf{I}$. The minimum distance $d_{\text {min }}$ normalized by the transmitted bit energy can be expressed as

$$
d_{\min }=\min _{\left(i_{1}, j_{1}\right) \neq\left(i_{2}, j_{2}\right)} \sqrt{\frac{1}{E_{b}}} D\left(\mathbf{S}_{\mathbf{I}^{\left(i_{1}\right)}}^{\left(j_{1}\right)}, \mathbf{S}_{\mathbf{I}^{\left(i_{2}\right)}}^{\left(j_{2}\right)}\right)
$$

where $E_{b}=E_{s}(N+L) / m=\left(E_{s}(N+L)\right) /\left(G\left(p_{I}+p_{M}\right)\right)$ is the average energy per bit and $E_{s}$ is the average symbol energy. Maximizing the MED between subblocks usually results in improved bit error rate (BER) performance. Therefore, when designing the constellation pair for subcarrier mapping in the ZTM-OFDM-IM, the MED between different subblocks 
should be greater than or equal to the MED between constellation points.

The constellation pair proposed in this paper is compared with the one in [14], where two different BPSK schemes shown in Figure 2 are adopted. The constellation A uses a pair of signal points located at $\{1,-1\}$, and the signal points of the constellation $\mathrm{B}$ are at $\{\sqrt{2} j,-\sqrt{2} j\}$, where $j=\sqrt{-1}$. In the proposed constellation pair shown in Figure 3, the constellation $\mathrm{B}$ exploits the signal points of 4-QAM, while the constellation A uses the same BPSK signal points.

Supposed that the number of subcarriers $N$, the length of CP $L$, and the maximum delay spread of the fading channel $v$ are set to 128,16 , and 10 , respectively. Assuming that the number of the disjoint group is 32 , there are four subcarriers in a subblock consisting of two active subcarriers and two inactive subcarriers. In the proposed ZTM-OFDM-IM system, one of the active subcarriers is modulated by the mapper $\mathrm{A}$ and the other is modulated by the mapper $\mathrm{B}$ as shown in Figure 3. However, in the previous system [14], three of the four subcarriers are activated, two of them are modulated by the mapper $\mathrm{A}$, and the other is modulated by the mapper B. The spectral efficiency of both systems is equal to 1.333 bit/s/Hz. According to (13), the normalized minimum distance of the proposed constellation pair shown in Figure 3 is $d_{\min }=2.667$, which is about $15.5 \%$ larger than $d_{\min }=2.309$ of the conventional constellation pair presented in Figure 2. Since $d_{\min }$ is one of the major factors for performance improvement, the proposed constellation pair possibly provides better BER performance. In Table 1, we compare the normalized MEDs of three index modulationbased OFDM systems with some specific parameters under the same spectral efficiency of $1.333 \mathrm{bit} / \mathrm{s} / \mathrm{Hz}$. It is considered that the ZTM-OFDM-IM with the proposed constellation pair has the largest $d_{\text {min }}$.

When the spectral efficiency is $2.222 \mathrm{bit} / \mathrm{s} / \mathrm{Hz}$, a constellation pair shown in Figure 4 has been used in the previous ZTMOFDM-IM system [14], where $a=(1+\sqrt{2}), b=-(1+\sqrt{2})$. The inner 4-QAM constellation is for the mapper A, and the outer 8 signal points are for the mapper $\mathrm{B}$, that is, $M_{A}=4$ and $M_{B}=8$, respectively. Let $d$ be the MED between a signal point in $\mathrm{A}$ and the nearest signal point in $\mathrm{B}$. In addition, the MED from the origin to any signal point in the constellation pair is also equal to $d$. Then, the Euclidean distances $d_{A}$ of the constellation $\mathrm{A}$ and $d_{B}$ of the constellation $\mathrm{B}$ are equal to $d_{A}=d_{B}=\sqrt{2} d$. Such constraints can be met with the new constellation pair presented in Figure 5. Both constellation pairs use the same signal set for the mapper A. However, the outer signal set for the mapper B in Figure 5 is different from the one in Figure 4. Nevertheless, both pairs have the same parameter set of $\left(n, k, k_{A}, k_{B}\right)=(4,3,2,1)$ for designing the ZTM-OFDM-IM system as given in Table 2 . It is noted that the proposed constellation pair has a larger normalized MED of 1.886 than the previous one. As presented in Table 2, the ZTM-OFDM-IM system with the proposed constellation pair has the largest $d_{\text {min }}$. It implicitly implies that the system with a new constellation pair may have slightly improved error performance.

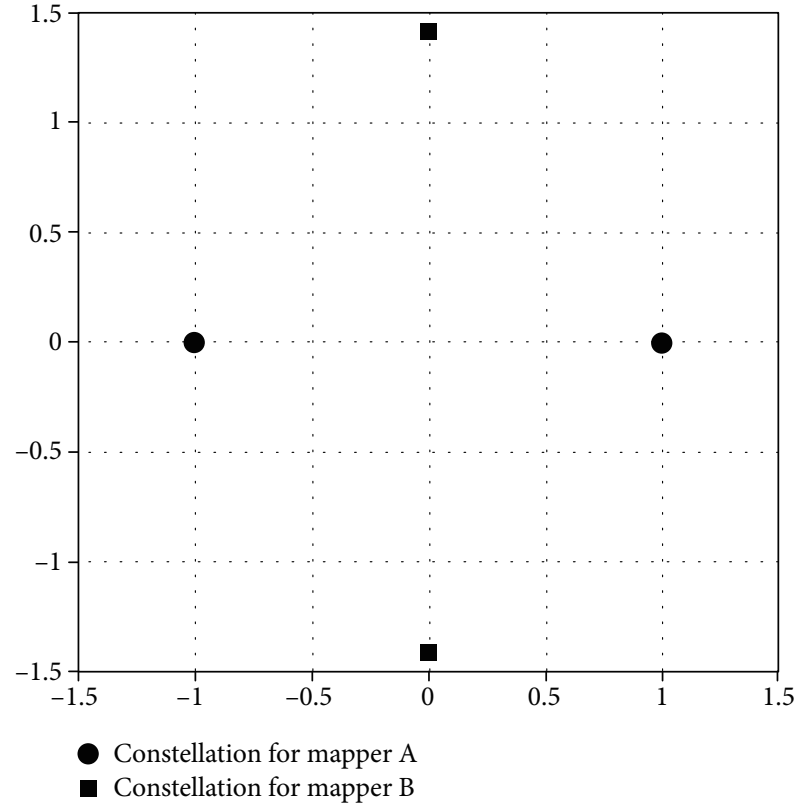

Figure 2: The constellation pair with $M_{A}=M_{B}=2$ in [14].

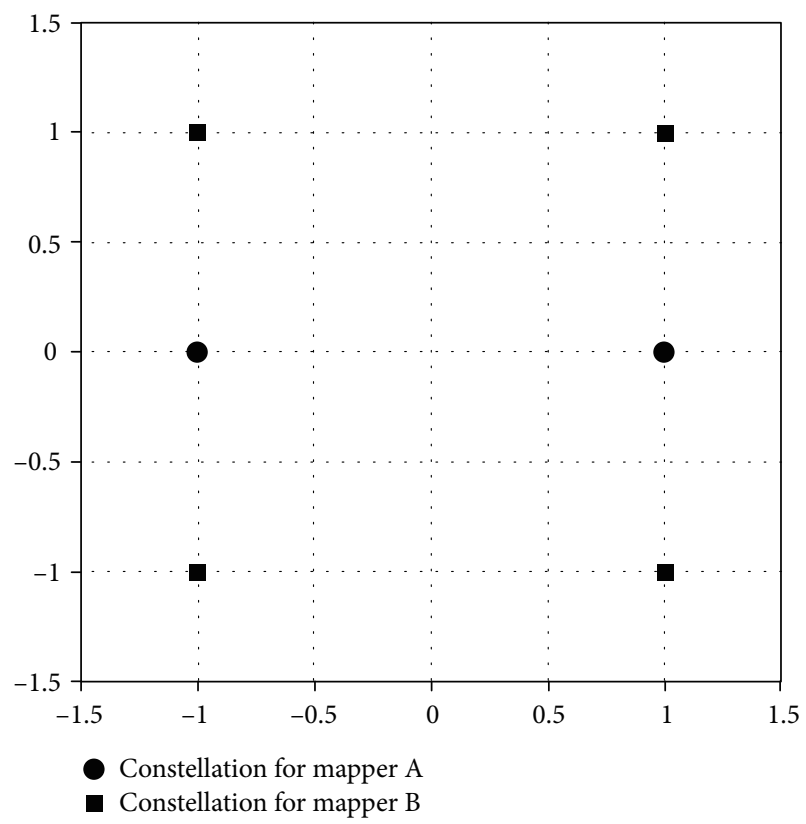

Figure 3: The new constellation pair with $M_{A}=2$ and $M_{B}=4$.

\section{Performance Analysis}

To evaluate performance of the proposed constellation pairs for the ZTM-OFDM-IM system, computer simulation has been carried out. The parameters presented in Tables 1 and 2 have been exploited to design the OFDM systems based on the IM scheme. We implement the frequency-selective Rayleigh fading channel as well as the AWGN channel using Matlab program as a transmission environment for simulation.

BERs of the OFDM systems with the IM scheme in the AWGN and frequency-selective Rayleigh fading channels are plotted in Figures 6 and 7 , respectively. Here, the 
TABLE 1: The parameters for IM schemes when $\eta=1.333 \mathrm{bit} / \mathrm{s} / \mathrm{Hz}$.

\begin{tabular}{lccccccc}
\hline Modulation & Constellation & $M_{A}$ & $M_{B}$ & $n$ & $k_{A}$ & $k_{B}$ & $P$ \\
\hline ZTM-OFDM-IM & Previous (Figure 2) & 2 & 2 & 4 & 2 & 1 & 6 \\
\hline DM-OFDM-IM & Proposed (Figure 3) & 2 & 4 & 4 & 1 & 1 & 6 \\
IM-OFDM-IM & $\{1,-1\},\{\sqrt{3} j,-\sqrt{3} j\}$ & 2 & 2 & 4 & 2 & 2.309 \\
\hline
\end{tabular}

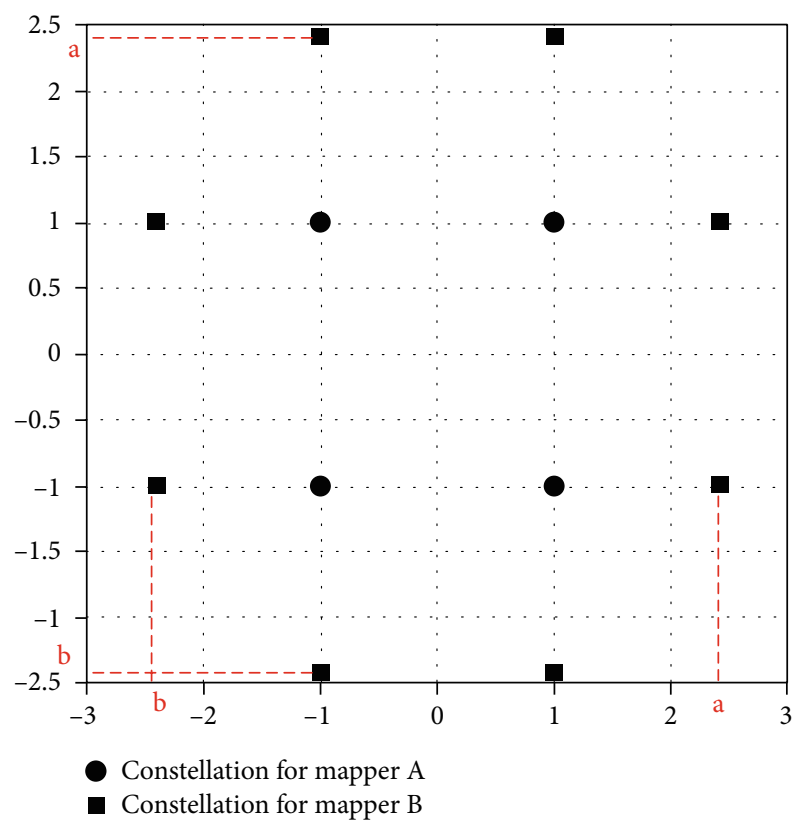

FIGURE 4: The previous constellation pair with $M_{A}=4$ and $M_{B}=8$.

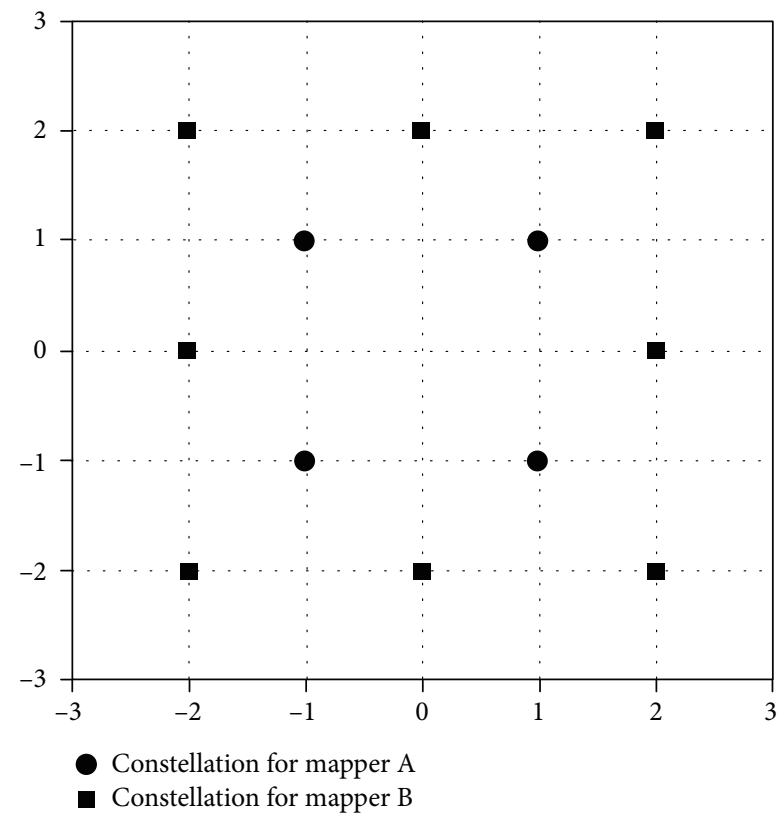

FIGURE 5: The new constellation pair with $M_{A}=4$ and $M_{B}=8$. parameters presented in Table 1 are used. To make the systems have the same spectral efficiency of $1.333 \mathrm{bit} / \mathrm{s} / \mathrm{Hz}$, the subcarriers in the previous ZTM-OFDM-IM system generated by the mapper A and the mapper B shown in Figure 2 are $k_{A}=2$ and $k_{B}=1$, respectively, while those in the proposed ZTM-OFDM-IM system produced by the mappers shown in Figure 3 are $k_{A}=k_{B}=1$. In the DM-OFDM-IM system, four subcarriers are divided into $k_{A}=k_{B}=2$, that is, no inactive subcarrier is exploited. In the case of the OFDMIM system, only one subcarrier is mapped using 16-QAM.

As a result, the ZTM-OFDM-IM system with the recommended constellation pair shows better error performance than the previous system. When the signal-to-noise ratio (SNR) is lower than $8.5 \mathrm{~dB}$ in the AWGN environment, it can be observed that the DM-OFDM-IM system has better error performance than other systems as shown in Figure 6. However, since BER is higher than $3.0 \times 10^{-4}$ in this case, it is considered that the DM-OFDM-IM is not suitable for application to a communication system. Specifically, for the BER of $10^{-6}$ as a reference, the proposed system has about $1.0 \mathrm{~dB}$ improvement in SNR over the conventional one. And the error performance of about $0.6 \mathrm{~dB}$ and $2.5 \mathrm{~dB}$ is improved compared to the DM-OFDM-IM and OFDM-IM systems, respectively. It is analyzed that such performance improvement of the proposed ZTM-OFDM-IM system is caused by the increased MED between subblocks.

In the Rayleigh fading channel, both ZTM-OFDM-IM systems have almost the same BER. However, it can be observed that those systems still have a performance gain of about $3.0 \mathrm{~dB}$ and $5.5 \mathrm{~dB}$, respectively, compared to the DM-OFDMIM and OFDM-IM systems at a reference BER of $10^{-5}$. It is due to the fact that the former systems have much increased MEDs compared to the latter systems as shown in Table 1.

When the spectral efficiency is increased to 2.222 $\mathrm{bit} / \mathrm{s} / \mathrm{Hz}$, the simulation results in the AWGN and Rayleigh fading channels are plotted in Figures 8 and 9 , respectively. Since the constellation pairs shown in Figures 4 and 5 are applied to the ZTM-OFDM-IM system, mapper $\mathrm{A}$ and mapper $\mathrm{B}$ are changed to $M_{A}=4$ and $M_{B}=8$, respectively, as presented in Table 2. In the DM-OFDM-IM system, mapper $\mathrm{A}$ and mapper $\mathrm{B}$ exploit the constellations shown in Figure 2, so $M_{A}=M_{B}=4$, and the OFDM-IM system generates subcarrier signals using 16-QAM.

Similar to Figure 6, the ZTM-OFDM-IM system with the proposed constellation pair has about $1.8 \mathrm{~dB}$ and $2.6 \mathrm{~dB}$ SNR gain in the AWGN channel, respectively, compared to the DM-OFDM-IM and OFDM-IM systems at the reference BER of $10^{-6}$. However, the difference in BERs of the previous and the new ZTM-OFDM-IM system is reduced to $0.3 \mathrm{~dB}$. 
TABLE 2: The parameters for IM schemes when $\eta=2.222 \mathrm{bit} / \mathrm{s} / \mathrm{Hz}$.

\begin{tabular}{lcccccccc}
\hline Modulation & Constellation & $M_{A}$ & $M_{B}$ & $n$ & $k_{A}$ & $k_{B}$ & $P$ & $d_{\min }$ \\
\hline \multirow{2}{*}{ ZTM } & Previous (Figure 4) & 4 & 8 & 4 & 2 & 1 & 10 & 1.812 \\
& Proposed (Figure 5) & 4 & 8 & 4 & 2 & 1 & 10 & 1.886 \\
\hline DM & Figure 2 in [13] & 4 & 4 & 4 & 2 & 2 & 10 & 1.371 \\
IM & $16-Q A M$ & $M=16$ & 4 & $k=2$ & 10 & 1.333 \\
\hline
\end{tabular}

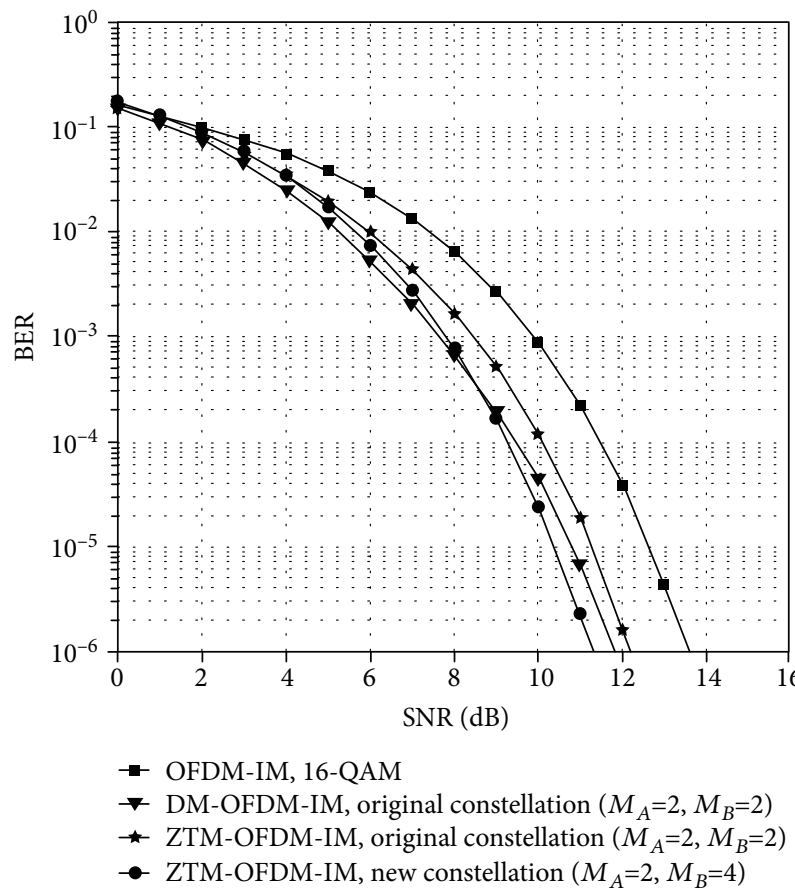

Figure 6: BERs in the AWGN channel when $\eta=1.333 \mathrm{bit} / \mathrm{s} / \mathrm{Hz}$.

As the MED gain of the new constellation pair over the previous pair decreases, the amount of performance improvement also decreases. The simulation results on the frequency-selective Rayleigh fading channel also show that the ZTM-OFDM-IM system with the new constellation pair has the best error performance as shown in Figure 9. Hence, maximizing the MED between different subblocks is crucial for improving performance of the OFDM system based on index modulation schemes.

\section{Conclusions}

This paper introduces new signal constellation pairs which improve the error performance of the ZTM-OFDM-IM system. In the presented system, one of the two mappers for mapping active subcarriers uses the same signal constellation as the one used in the previous work, and the other exploits a constellation larger than the one used in [14]. This causes the MED between the subblocks of the ZTM-OFDM-IM system with new constellation pairs to be increased under the constraint of the same spectral efficiency. It is a major factor to improve the bit error performance of the proposed system.

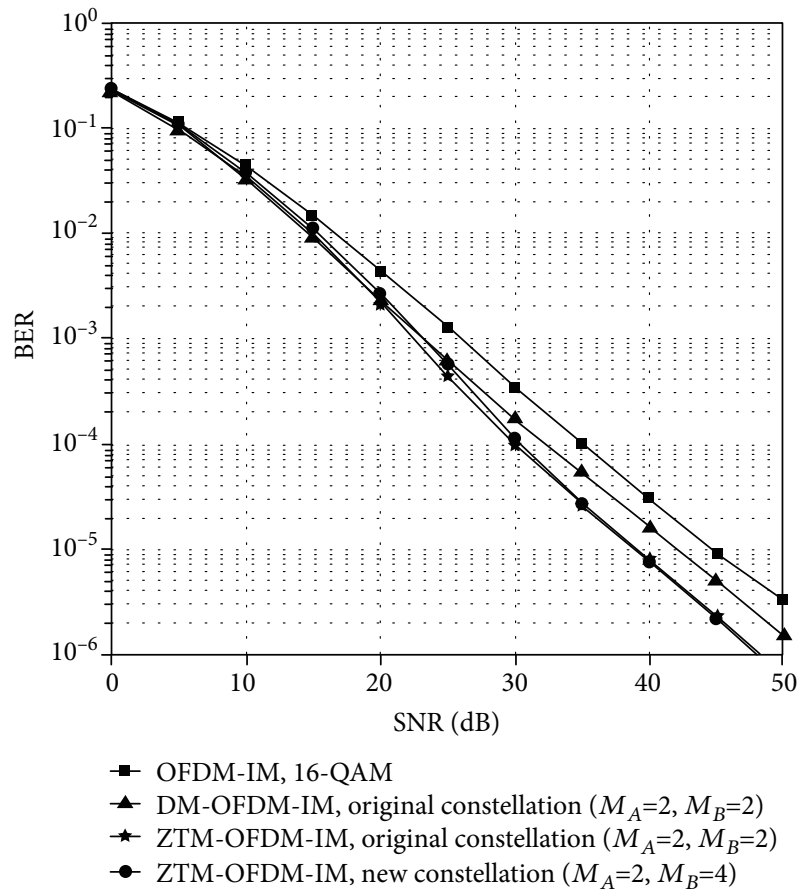

FIGURE 7: BERs in the Rayleigh fading channel when $\eta=1.333$ $\mathrm{bit} / \mathrm{s} / \mathrm{Hz}$.

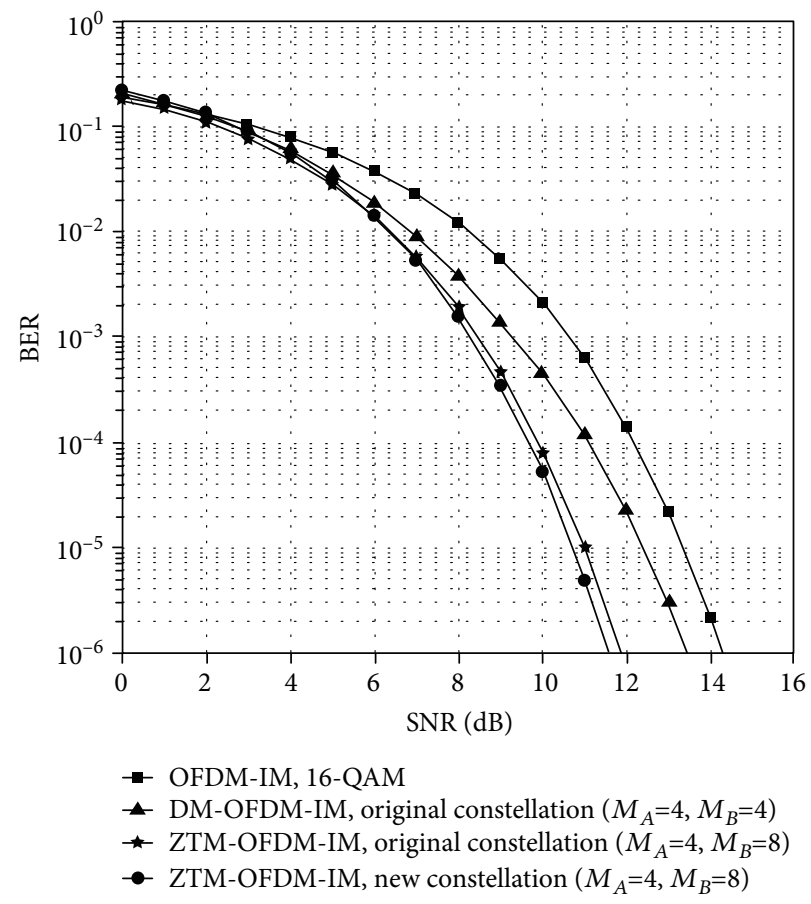

Figure. 8: BERs in the AWGN channel when $\eta=2.222 \mathrm{bit} / \mathrm{s} / \mathrm{Hz}$.

As a result of simulation in ideal AWGN and frequencyselective Rayleigh fading channel environments, the ZTMOFDM-IM system with the proposed constellation pairs has much lower BER than the typical OFDM system with IM scheme such as OFDM-IM and DM-OFDM-IM. In addition, the new ZTM-OFDM-IM system shows slightly better 


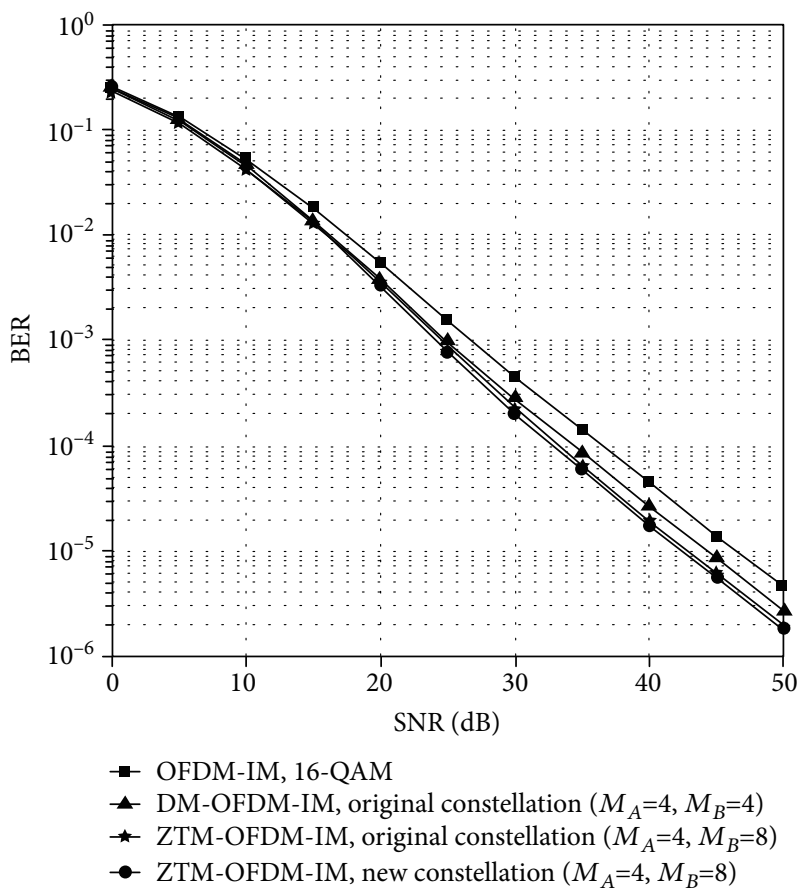

FIGURE 9: BERs in the Rayleigh fading channel when $\eta=2.222$ $\mathrm{bit} / \mathrm{s} / \mathrm{Hz}$.

performance than the same system with the conventional constellation pairs. The new constellation pairs proposed in this paper increase the MED between subblocks, demonstrating the possibility of improving error performance of the ZTM-OFDM-IM system. Hence, it is considered that a further study on generalized design of the constellation pairs for the mappers of the ZTM-OFDM-IM system is necessary in the future. In addition, another investigation on the IMbased OFDM system is necessary to further improve spectral and energy efficiencies while suppressing system complexity.

\section{Data Availability}

The data used to support the findings of this study are available from the corresponding author upon request.

\section{Conflicts of Interest}

The authors declare that there are no conflicts of interest regarding the publication of this paper.

\section{References}

[1] J. Joung, C. K. Ho, and S. Sun, "Spectral efficiency and energy efficiency of OFDM systems: impact of power amplifiers and countermeasures," IEEE Journal on Selected Areas in Communications, vol. 32, no. 2, pp. 208-220, 2014.

[2] F. Adib and D. Katabi, "See through walls with Wi-Fi!," ACM SIGCOMM Computer Communication Review, vol. 43, no. 4, pp. 75-86, 2013.

[3] D. Astely, E. Dahlman, A. Furuskär, Y. Jading, M. Lindström, and S. Parkvall, "LTE: the evolution of mobile broadband,"
IEEE Communications Magazine, vol. 47, no. 4, pp. 44-51, 2009.

[4] A. Ghosh, D. R. Wolter, J. G. Andrews, and R. Chen, "Broadband wireless access with WiMax/802.16: current performance benchmarks and future potential," IEEE Communications Magazine, vol. 43, no. 2, pp. 129-136, 2005.

[5] A. Peled and A. Ruiz, "Frequency domain data transmission using reduced computational complexity algorithms," in ICASSP '80. IEEE International Conference on Acoustics, Speech, and Signal Processing, vol. 5, pp. 964-967, Denver, Colorado, USA, USA, Apr. 1980.

[6] E. Basar, M. Wen, R. Mesleh, M. Di Renzo, Y. Xiao, and H. Haas, "Index modulation techniques for next-generation wireless networks," IEEE Access, vol. 5, pp. 16693-16746, 2017.

[7] T. Mao, Q. Wang, Z. Wang, and S. Chen, "Novel index modulation techniques: a survey," IEEE Communications Surveys \& Tutorials, vol. 21, no. 1, pp. 315-348, 2019.

[8] R. Abu-Alhiga and H. Haas, "Subcarrier-index modulation OFDM," in 2009 IEEE 20th International Symposium on Personal, Indoor and Mobile Radio Communications, pp. 177181, Tokyo, Japan, Sep. 2009.

[9] D. Tsonev, S. Sinanovic, and H. Haas, "Enhanced subcarrier index modulation (SIM) OFDM," in 2011 IEEE GLOBECOM Workshops, pp. 728-732, Houston, TX, USA, Dec. 2011.

[10] E. Basar, U. Aygolu, E. Panayirci, and H. V. Poor, "Orthogonal frequency division multiplexing with index modulation," IEEE Transactions on Signal Processing, vol. 61, no. 22, pp. 5536$5549,2013$.

[11] M. Wen, B. Ye, E. Başar, Q. Li, and F. Ji, "Enhanced orthogonal frequency division multiplexing with index modulation," IEEE Transactions on Wireless Communications, vol. 16, no. 7, pp. 4786-4801, 2017.

[12] R. Fan, Y. J. Yu, and Y. L. Guan, "Generalization of orthogonal frequency division multiplexing with index modulation," IEEE Transactions on Wireless Communications, vol. 14, no. 10, pp. 5350-5359, 2015.

[13] T. Mao, Z. Wang, Q. Wang, S. Chen, and L. Hanzo, "Dualmode index modulation aided OFDM," IEEE Access, vol. 5, pp. 50-60, 2017.

[14] T. Mao, Q. Wang, J. Quan, and Z. Wang, "Zero-padded orthogonal frequency division multiplexing with index modulation using multiple constellation alphabets," IEEE Access, vol. 5, pp. 21168-21178, 2017. 